\title{
Loss and load reduction by coordinated control of community energy storage and OLTC
}

\author{
Jae Won Chang, Ki Hoon Kang, Gyu Sub Lee, Jin Oh Lee, Seung-Il Moon ${ }^{\text {a* }}$ \\ Department of Electrical and Computer Engineering, Seoul National Univ. \#013, 1 Gwanangno, Gwanak-gu, Seoul, 08826, \\ South Korea
}

\begin{abstract}
Renewable energy sources are usually integrated with a distribution system through a small-size-generator with low voltage level. As a consequence, distributed energy storage systems have been connected to a distribution system to help with renewable energy resources integration. Community Energy Storage (CES) is a small distributed energy storage connected to the secondary part of a transformer and is an advanced energy storage system close to the customer. CES provides many benefits such as peak shaving, load shifting and voltage control. Meanwhile, distribution system operators conduct Conservation Voltage Reduction (CVR) to reduce power consumption in the system. To implement CVR on the system, flat voltage profile is required. CES is distributed in the distribution system and is capable of controlling system voltage. In this paper, a control method of CES is proposed in order to implement CVR. Based on day-ahead load forecasting, power consumption including load demand and active power loss is reduced by the optimal schedule of CES output power and On-Load Tap-Changer (OLTC) operation. With a case study using Matpower, this paper validates the effect of the proposed method.
\end{abstract}

Keywords: Community energy storage, conservation voltage reduction, loss reduction, load reduction, OLTC

\section{Introduction}

Nomenclature

$E_{\text {grid }}$ : Primary bus voltage of transformer

$E_{S}:$ Sending part voltage of distribution system

$E_{R}:$ Receiving part voltage of distribution system $E_{C}:$ Bus voltage connected with CES

$P_{\text {grid }}, Q_{\text {grid }}$ : Active/reactive power from transmission system to distribution system

$P_{\text {load }}, Q_{\text {load }}:$ Load demanding active/reactive power

$P_{R}, Q_{R}:$ Active/reactive power from sending end to receiving end

$P_{C E S}, Q_{C E S}$ : Exchanged active/reactive power with CES

$R_{L}, X_{L}$ : Equivalent resistor/reactance of distribution line

$X_{T r}$ : Equivalent impedance of transformer

$\gamma:$ Turn ratio of transformer

$P_{\text {Loss }}^{h}:$ Power loss of system at time $\mathrm{h}$

$\Phi$ : Grid angle

$V_{\max }, V_{\min }:$ Maximum and minimum voltage of system allowance $P_{C E S, i}^{h}:$ Active power of CES i at time $\mathrm{h}$

tap $_{\max }$, tap $_{\min }$ : Maximum and minimum of tap position

$V_{k}^{h}:$ Voltage of bus $\mathrm{k}$ at time $\mathrm{h}$ $\operatorname{tap}^{h}$ : Tap position of OLTC at time $\mathrm{h}$

\footnotetext{
* Manuscript received December 1, 2015; revised April 2, 2016.

Corresponding author. Tel.: +82-10-5001-6600; E-mail address: jwchang91@gmail.com

doi: $10.12720 /$ sgce.5.2.86-93
} 


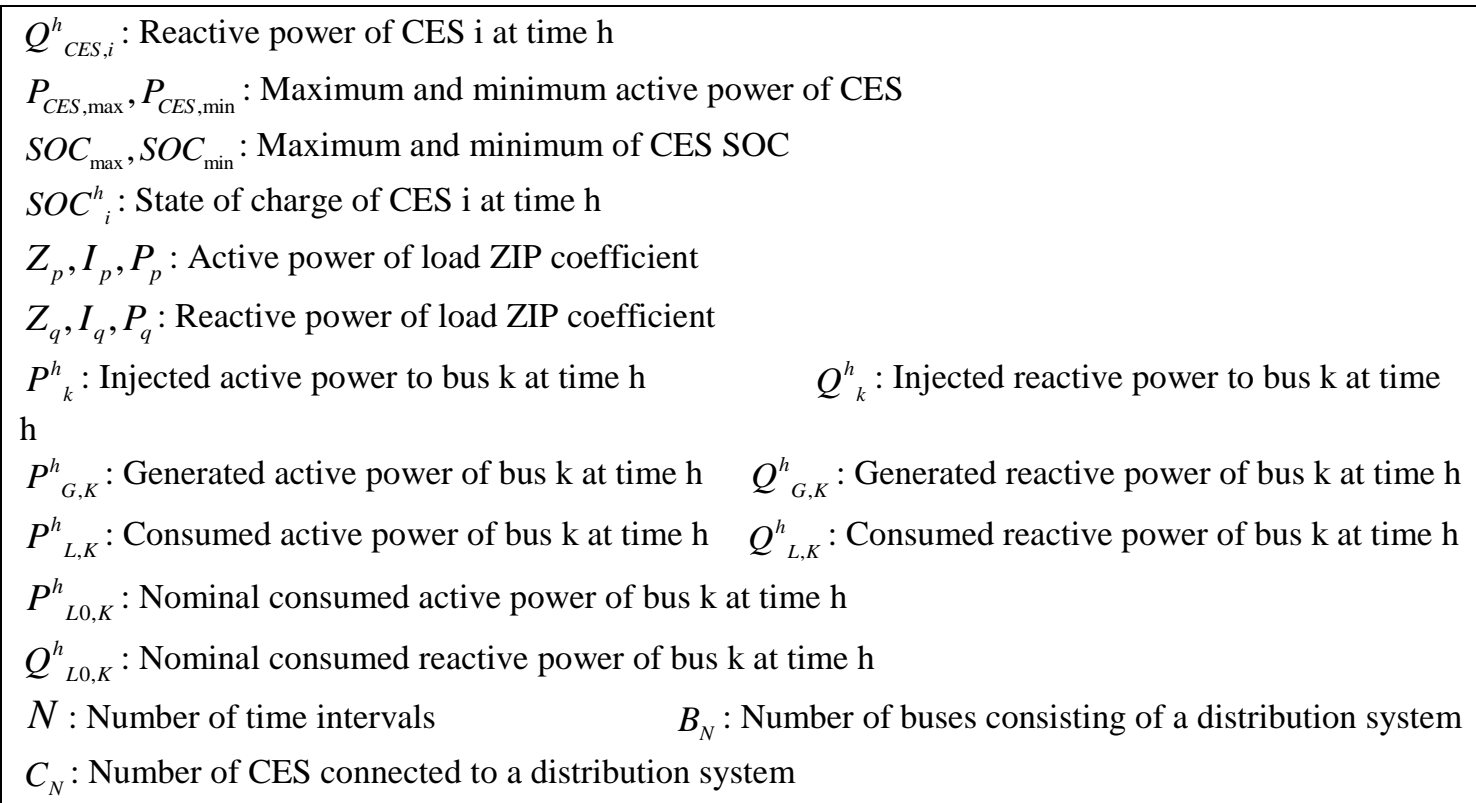

Renewable Energy Sources (RES) are directly integrated into a distribution system because they are small and the output voltage is low [1]. An Energy Storage System (ESS) is integrated with a distribution system to mitigate the output of RES. A distributed ESS is also used to control grid voltage and supply the reserve for frequency regulation [2]-[3].

Recently, ESS is installed similar to Community Energy Storage (CES) due to the small scale of RES, which is close to the consumer such as a domestic Photovoltaic (PV) panel [4]. CES is a type of ESS, which has small capacity and is connected to the secondary part of a distribution transformer. Because CES is located near the customer, reliability of CES is more credible and efficient than the reliability of ESS. CES is than ESS. In addition, CES is widely spread and has easy to control voltage with small capacity. Thus, CES is cheap to maintain and has a minor influence on the grid [5]-[7]. The operation of CES for reducing peak load in a distribution system integrated with PV generation and optimization of CES output for load leveling is presented in [8], [9]. In [10], using predicted load and price information, a method for maximizing operational advantage of CES is used to achieve an economic profit. Also, minimizing the fluctuation of system voltage due to intermittent PV generation with CES is proposed [11].

This paper proposes CES could be used for CVR. CVR is a method that reduces the whole active power of a load maintaining a low voltage level with a range of allowance. The earlier versions of CVR use a Step Voltage Regulator (SVR) for the feeder, shunt capacitor (ShC) and On-Load Tap-Changer (OLTC) of the transformer to flatten the voltage of the system. CES is distributed to a system and can control voltage by using output controlling. Thus, flattening voltage using CES could be used for CVR.

In this paper, we consider CES integrated with a distribution system of which voltage is controlled by the OLTC and CES which reduces a voltage of a connected point of the system through active/reactive power. Therefore, we propose coordinated control for reducing the loss and load power with CVR. The voltage for each bus, and all scheduling intervals for the State of Charge (SOC) and output must be kept within a range of constraints. The interior point solver of MATPOWER is used for the simulation results. This paper is organized as follows. Section 2 provides theoretical analysis of CES output control. Section 3 proposes CES output scheduling. Section 4 provides simulation and experimental results. Section 5 is the conclusions.

\section{Theoretical Analysis of CES Output Control}

In this section, a system voltage equation is derived when CES is connected with a distribution system. 
Also, we verified that the control of active/reactive power of CES can control system voltage and loss.

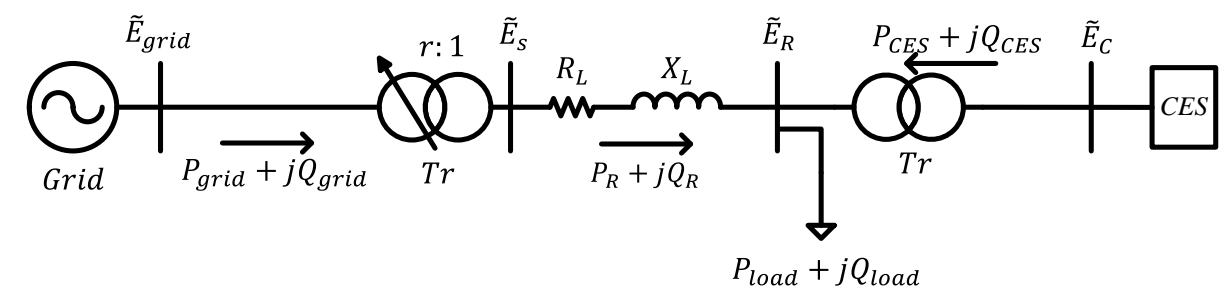

Fig. 1. CES connected to radial distribution system.

\subsection{System voltage control and loss using CES output}

Fig. 1 shows a simplified distribution system for theoretical analysis. OLTC and CES can be used to control system voltage. CES also controls system voltage for controlling active/reactive power. We assume that system voltage is near rated voltage and that CES can supply/absorb active/reactive power as operator instructions. Also, we assume that the loads consist of a constant power model and the cooper loss of the transformer can be ignored.

The receiving part, sending part voltage and power loss were represented by the following equations:

$$
\begin{aligned}
& E_{R}=\sqrt{\frac{-B \pm \sqrt{B^{2}-4 A C}}{2 A}} \\
& A=1, B=-E_{S}{ }^{2}+-2\left(R_{L} P_{R}+X_{L} Q_{R}\right), C=\left(R_{L}{ }^{2}+X_{L}{ }^{2}\right)\left(P_{R}{ }^{2}+Q_{R}{ }^{2}\right) \\
& E_{S}=\sqrt{\left(\frac{E_{\text {grid }}^{2}}{r}\right)^{2}-\frac{2 E_{\text {grid }}}{r} \frac{X_{T r}}{E_{R}}\left(Q_{R} \cos \Phi+P_{R} \sin \Phi\right)+\left(\frac{X_{T r}}{E_{R}}\right)^{2}\left(P_{R}{ }^{2}+Q_{R}{ }^{2}\right)} \\
& P_{\text {loss }}=\left|I_{R}\right|^{2} R_{L}=\left|\frac{P_{R}-j Q_{R}}{E_{R}}\right|^{2} R_{L}, P_{R}+j Q_{R}=\left(P_{\text {load }}+P_{C E S}\right)+j\left(Q_{\text {load }}+Q_{C E S}\right)
\end{aligned}
$$

$P_{R}$ and $Q_{R}$ were determined by $P_{C E S}$ and $Q_{C E S}$, respectively. Consequently, voltage and active power of the sending part and receiving part were decided by $P_{C E S}, Q_{C E S}$ and the tap position of the OLTC in the following equations:

$$
\begin{aligned}
& E_{R}=f_{1}\left(P_{R}, Q_{R}\right)=f_{2}\left(P_{C E S}, Q_{C E S}, \text { tap }\right) \\
& E_{S}=g_{1}\left(P_{R}, Q_{R}\right)=g_{2}\left(P_{C E S}, Q_{C E S}, \text { tap }\right) \\
& P_{\text {loss }}=h_{1}\left(P_{R}, Q_{R}\right)=h_{2}\left(P_{C E S}, Q_{C E S}, \text { tap }\right)
\end{aligned}
$$

\section{CES Output Scheduling}

Scheduling with OLTC and CES is performed using Matpower [12]. For optimal scheduling, the objective function and constraints have to be determined. 


\subsection{Objective function}

The object of this paper is that when a load is changed with time in a distribution system connected with CES, power loss and load power are reduced to maintain the system voltage level with the proper range. Unlike an earlier CVR operation using OLTC, SVR and ShC, the output power control method using CES with OLTC is proposed. The sum of load power and loss is the objective function.

$$
J=\operatorname{Min}\left(\sum_{h=1}^{N} \sum_{k=1}^{B_{N}} P_{L, k}^{h}+P_{\text {Loss }}^{h}\right)
$$

\subsection{Constraints}

There are several constraints for finding the optimal solution to an objective function.

First, system voltage and OLTC have constraints based on the operation of the distribution system.

$$
\begin{aligned}
& V_{\min } \leq V_{k}^{h} \leq V_{\max } \text { for } h=1,2, \cdots, N \text { and } k=1,2, \cdots, B_{N} \\
& \operatorname{tap}_{\min } \leq \operatorname{tap}^{h} \leq \operatorname{tap}_{\max } \text { for } h=1,2, \cdots, N
\end{aligned}
$$

The output and SOC of the CES are given by the manufacturer.

$$
\begin{aligned}
& P_{C E S, \min } \leq P_{C E S, i}^{h} \leq P_{C E S, \max } \text { for } h=1,2, \cdots, N \text { and } i=1,2, \cdots, C_{N} \\
& S O C_{\text {min }} \leq S O C_{i}^{h} \leq S O C_{\text {max }} \text { for } h=1,2, \cdots, N \text { and } i=1,2, \cdots, C_{N}
\end{aligned}
$$

The maximum and minimum reactive power of CES is determined by $S_{C E S \text {, max }}$ and $P_{C E S}$ :

$$
-\sqrt{S_{C E S, \max }^{2}-P_{C E S, i}^{h}} \leq Q_{C E S, i}^{h} \leq-\sqrt{S_{C E S, \max }^{2}-P_{C E S, i}^{h}}
$$

for $h=1,2, \cdots, N$ and $i=1,2, \cdots, C_{N}$

The active power of CES and the SOC of CES are expressed as:

$$
S O C_{i}^{h+1}-S_{i} O C_{i}^{h}+P_{C E S, i}^{h} \times c=0 \text { for } h=1,2, \cdots, N \text { and } i=1,2, \cdots, C_{N}
$$

For the next period of scheduling, the end and start values of the SOC are the same.

$$
\operatorname{SOC}_{i}^{1}-\operatorname{SOC}_{i}^{N+1}=0 \text { for } i=1,2, \cdots, C_{N}
$$

The buses are not connected with the CES and have constraints.

$$
\begin{gathered}
P_{k}^{h}-P_{G, k}^{h}+P_{L, k}^{h}=0 \text { for } h=1,2, \cdots, N \text { and } C E S \notin k \\
Q_{k}^{h}-Q_{G, k}^{h}+Q_{L, k}^{h}=0 \text { for } h=1,2, \cdots, N \text { and } C E S \notin k
\end{gathered}
$$

The buses are connected with the CES and also have constraints. 


$$
\begin{aligned}
& P_{k}^{h}-P_{G, k}^{h}+P_{L, k}^{h}-P_{C E S, i}^{h}=0 \text { for } h=1,2, \cdots, N \text { and } C E S \notin k \\
& Q_{k}^{h}-Q_{G, k}^{h}+Q_{L, k}^{h}-Q_{C E S, i}^{h}=0 \text { for } h=1,2, \cdots, N \text { and } C E S \notin k
\end{aligned}
$$

For applying CVR, there are constraints for the system load model.

$$
\begin{aligned}
& P_{L, k}^{h}-P_{L 0, k}^{h}\left(Z_{p}\left(\frac{V_{k}^{h}}{V_{0}}\right)^{2}+I_{p} \frac{V_{k}^{h}}{V_{0}}+P_{p}\right)=0 \text { for } h=1,2, \cdots, N \text { and } k=1,2, \cdots, B_{N} \\
& Q_{L, k}^{h}-Q^{h}{ }_{L 0, k}\left(Z_{q}\left(\frac{V_{k}^{h}}{V_{0}}\right)^{2}+I_{q} \frac{V_{k}^{h}}{V_{0}}+P_{q}\right)=0 \text { for } h=1,2, \cdots, N \text { and } k=1,2, \cdots, B_{N}
\end{aligned}
$$

\subsection{Matpower for CES output scheduling}

The interior point solver from Matpower is used to draw optimal coordinated scheduling, which satisfy the above objective function with constraints. The variables used for the interior point solver are

$$
x=\left[P_{C E S, i}^{h}, Q_{C E S, i}^{h}, S O C_{i}^{h}, \operatorname{tap}^{h}, V_{k}^{h}, \theta_{k}^{h}, P_{G, k}^{h}, Q_{G, k}^{h}, P_{L, k}^{h}, Q_{L, k}^{h}\right]^{T}
$$

\section{Case Study}

For verifying a reduction of load power and power loss, a distribution system is formed based on the IEEE 13 test bus system. The simulation system is composed of 13 buses and 32 tap positions of the OLTC. The OLTC controls the voltage of Bus 2 in order to regulate the voltage of the whole distribution system. On Buses 5, 12 and 13, a PV generator and CES are connected. Four of the CES are connected with the secondary part of a distribution transformer. The capacity and maximum output are based on [13]. The initial SOC state is 0.6 and the SOC is maintained from 0.3 to 0.9 for the life cycle. The maximum output of the PV generator is 0.1MW. Simulation system is shown in Fig. 3.
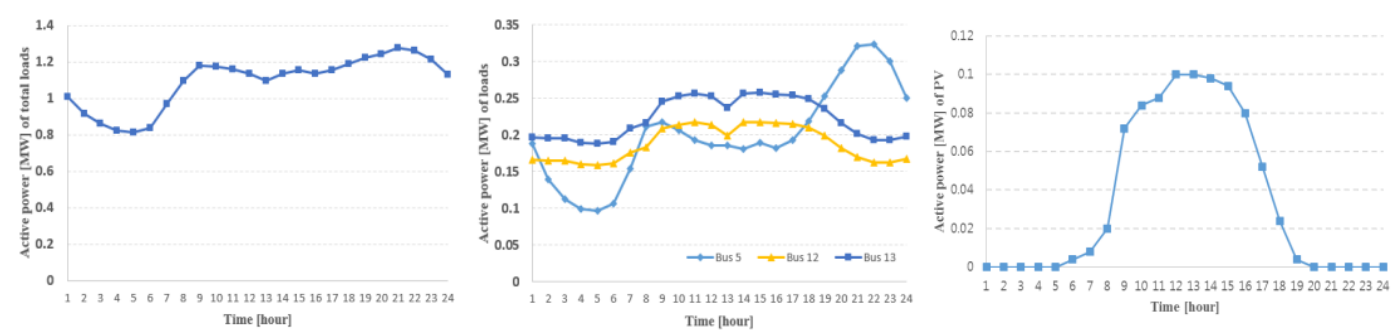

Fig. 2. Active power of total loads, load on Buses 5, 12 and 13, and PV generator.

Table 1. Parameters of the simulation system

\begin{tabular}{l|l}
\hline Distribution lines & Modified IEEE 13-bus test system \\
\hline Transformer & $115 / 4.16 \mathrm{kV}, 5 \mathrm{MVA}, \mathrm{x}=8 \%, \mathrm{r}=1 \%$ \\
\hline OLTC & At $\mathrm{HV}$ side, $-10 \%$ to $10 \%$ regulation with 32 steps \\
\hline Loads & ZIP_residential $: \mathrm{Zp}=1.5, \mathrm{Ip}=-2.31, \mathrm{Pp}=1.81$ \\
& $\mathrm{Zq}=7.41, \mathrm{Iq}=-11.97, \mathrm{Pq}=5.55$ \\
& ZIP_industrial $: \mathrm{Zp}=1.21, \mathrm{Ip}=-1.61, \mathrm{Pp}=1.41$ \\
& $\mathrm{Zq}=4.35, \mathrm{Iq}=-7.08, \mathrm{Pq}=3.72$ \\
\hline CESs & Rated power $: 25 \mathrm{~kW}$ \\
& Capacity $: 50 \mathrm{kWh}$ \\
& Efficiency $: 100 \%$ \\
& Maximum SOC $: 0.9$ \\
& Minimum SOC $: 0.3$ \\
& Initial SOC $: 0.6$ \\
\hline
\end{tabular}


We assume that load prediction is performed every hour and the load is comprised of constant power, constant current, and constant impedance load. ZIP coefficient for Industrial load and residential load is based on [14]. The parameters are shown in Table 1. Also, load and PV information is shown in Fig. 2.

The load consists of an industrial load and a residential load. Bus 5 is connected with the CES as a residential bus. Buses 12 and 13 are connected with the CES as an industrial load.

Two cases are compared for verification of the study. Case 1 is the multi CESs connected system. Case 2 is the control for the system voltage using the coordinated control of OLTC and a single ESS connected to a distribution system. Capacity and maximum output of a single ESS is the same as the capacity and maximum output of a whole CESs. Location of ESS is decided as Bus 13, which can mostly decrease the consumed power and loss branch. Case 1 and case 2 of the simulation systems are shown in Fig. 3.

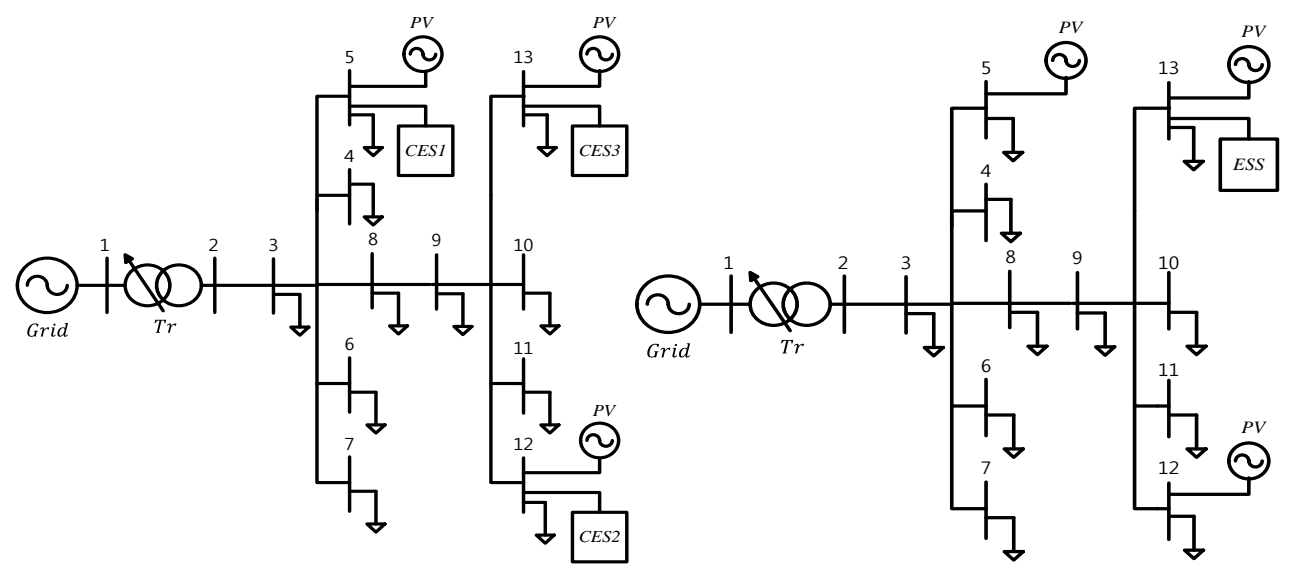

Fig. 3. Modified IEEE 13 test bus (multi CES connected system and single ESS connected system).

Table 2. Scheduling results for Case 1 and Case 2

\begin{tabular}{l|l|l|l|l}
\hline Case & Case 1 & Case 2 & Case1 - Case2 & (Case1-Case2)/Case1 [\%] \\
\hline Total load[MW] & 26.225 & 26.193 & 0.03 & 0.114 \\
\hline Loss[MW] & 0.438 & 0.417 & 0.02 & 4.566 \\
\hline Total load+Loss[MW] & 26.663 & 26.62 & 0.053 & 0.199 \\
\hline
\end{tabular}

Table 2 shows the optimal scheduling results for Case 1 and Case 2. In the situation where multi CESs are connected to a distribution system, the consumed power is lower than the consumed power of a single ESS connected case. The total load, loss, and sum of total load and loss fell by $0.114 \%, 4.56 \%$, and $0.199 \%$, respectively.
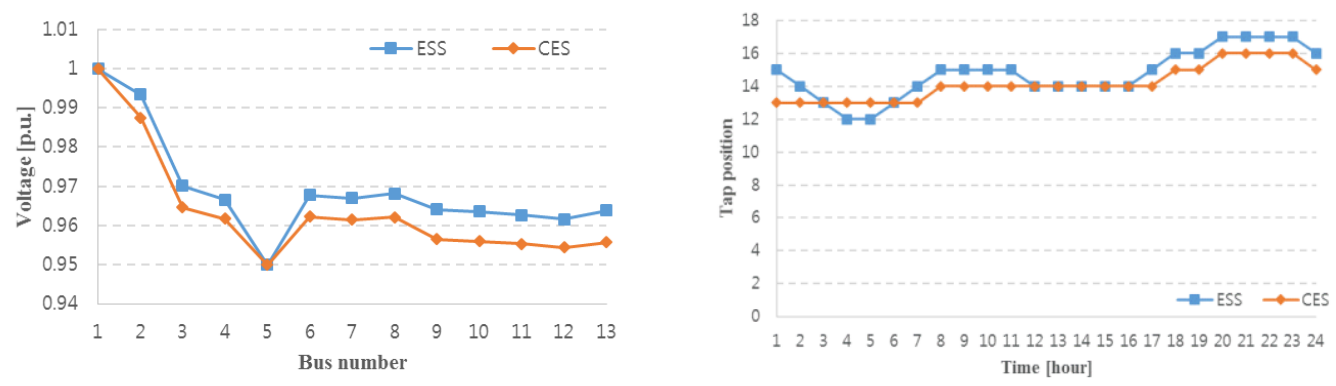

Fig. 4. Voltage of each bus at the heaviest load time and the tap position of the OLTC.

Fig. 4 shows that the system voltage of the OLTC and multi connected CESs is lower than the voltage of single connected ESS at the heaviest loading time. It is impossible to change to a lower tap position because the voltage of Bus 5 is at a minimum tolerance range. As ESS is connected to bus 13, it is 
difficult to compensate for the voltage of Bus 5. However, as in the multi CESs connected cases, CES connected with Bus 5 can compensate for the voltage of Bus 5. The tap position of the OLTC can also be low and the system voltage can be maintained low.

Fig. 4 also shows the tap position of the OLTC for the two cases. The tap position for multi CESs connected cases is lower than the tap position of a single ESS connected case for most of the time. Because the distribution of CES in the system compensates each voltage of the bus effectively, system voltage can be low and the tap position of the OLTC can be low.

\section{Conclusions}

ESS is distributed in a distribution system in order to directly connect the RES with a distribution system for mitigating the output of RES. Distributed ESS not only mitigates the output of RES, but also affects the control of system voltage, frequency regulation and demand response. Recently, the RES is closed to customers. ESS is also installed as a CES near the customer. CES takes a small load and is installed on a secondary part of a distribution transformer. CES is available to reduce the peak load, system operation cost and control system voltage. Earlier versions of CVR control system voltage with OLTC, SVR, and ShC. Because CES is distributed in a system and can control system voltage using output control, we notice that CES could also be used for CVR.

This paper proposed a coordinated control method of OLTC and CES output for CVR, through ability of reducing the voltage of an integrated branch in order to decrease consumed power. In other words, based on the predicted load, schedule of active/reactive power of CES output and the tap position of OLTC, load power and loss could be reduced. For scheduling derivation, this paper used the interior point solver provided by Matpower.

Also, the proposed control method was verified by theoretical analysis and a case study was performed. The case study showed that the proposed control method improved the reduction of load power and loss power in comparison to a previous control method.

Consequently, the proposed control method showed that CES could be used for renewable energy resources integration and could improve system operation efficiency through CVR.

\section{Acknowledgements}

This work was supported by the Global Excellent Technology Innovation (20132010101890) of the Korea Institute of Energy Technology Evaluation and Planning(KETEP), granted financial resource from the Ministry of Trade, Industry \& Energy, Republic of Korea.

\section{References}

[1] Freris L, Infield D. Renewable Energy in Power System, John Wiley \& Sons; 2008.

[2] Stadler M, Kloess M, Groissbock M, Cardoso G, Sharma R, Bozchalui MC, et al., Electric storage in California's commercial buildings. Applied Energy, 2013; 104:711-722.

[3] Schroeder A. Modeling storage and demand management in power distribution grids. Applied Energy, 2011; 88:4700-4712.

[4] Jung J, Cho Y, Cheng D, Onen A, Arghandeh R, Dilek M, et al., Monte Carlo analysis of plug-in hybrid vehicles and distributed energy resource growth with residential energy storage in Michigan. Applied Energy, 2013; 108:218-235.

[5] Nourai A, Sastry R, Walker T. A vision \& strategy for deployment of energy storage in electric utilities. Power and Energy Society General Meeting, 2010:1-4.

[6] Parra D, Gillott M, Norman SA, Walker GS. Optimum community energy storage system for PV energy time-shift. Applied Energy, 2015; 137:576-587.

[7] Zhu W, Garrett D, Butkowski J, Wang Y. Overview of distributed energy storage systems for residential communities. Energytech, 2012:1-6.

[8] Huq KMM, Baran ME, Lukic S, Nare OE. An energy management system for a community energy storage system. Energy Conversion Congress and Exposition, 2012: 2759-2763.

[9] Meng F, Haughton D, Chowdhury B, Crow ML, Heydt GT. Distributed generation and storage optimal control with state estimation. IEEE Transaction on Smart Grid, 2013; 4(4):2266-2273. 
[10] Arghandeh R, Woyak J, Onen A, Jung J, Broadwater RP. Economic optimal operation of community energy storage systems in competitive energy markets. Applied Energy, 2014; 135:71-80.

[11] Sugihara H, Yokoyama K, Saeki O, Tsuji K, Funaki T. Economic and efficient voltage management using customer-owned energy storage systems in a distribution networks with high penetration of photovoltaic systems. IEEE Transactions on Power Systems, 2012; 28(1):102-111.

[12] Zimmerman RD, Murillo-Sanchez C. Matpower User's Manual. [Online]. Available: http://www.pserc.cornell.edu/matpower

[13] Wang Z, Wang J. Review on implementation and assessment of conservation voltage reduction. IEEE Transaction on Power Systems, 2014; 29(3):1306-1315.

[14] Bokhari A, Alkan A, Dogan R, Diaz-Aguilo M, et al., Experimental determination of the ZIP coefficients for modern residential, commercial, and industrial loads. IEEE Transactions on Power Delivery, 2013; 29(3):1372-1381. 